\title{
TAGUNG
}

\section{Ein Geschenk für die Integrationsforschung? Facetten und Wandel des Europäischen Rates}

\author{
Tobias Kunstein und Wulf Reiners*
}

Neben der zentralen Rolle des Europäischen Rates in Zeiten der Finanz- und Staatsschuldenkrise und neuer außenpolitischer Herausforderungen stand auch ein mehrfaches Jubiläum Pate bei der Themenauswahl zu dieser Tagung: Wolfgang Theodor Wessels, JeanMonnet-Professor an der Universität zu Köln, beging im Jahr 2013 nicht nur sein zwanzigjähriges Dienstjubiläum und sein 40. Jahr im Institut für Europäische Politik, sondern auch seinen 65. Geburtstag. Mit Blick auf das besondere Interesse des Jubilars für den Forschungsgegenstand versammelten die Organisatoren eine Reihe von namhaften Rednern aus Wissenschaft und Praxis, um den Europäischen Rat als das Schlüsselorgan der Europäischen Union in dreierlei Hinsicht zu beleuchten: (1.) in seiner historischen Entwicklung; (2.) als weltpolitischen Akteur; und (3.) als Wirtschaftsregierung der Europäischen Union. Der Teilnehmerkreis der Konferenz setzte sich aus Nachwuchswissenschaftlern, Studierenden, Professoren und Experten für die institutionelle Entwicklung des EU-Systems zusammen. ${ }^{1}$

\section{Die Entwicklung des Europäischen Rates}

Die Evolution des Europäischen Rates, von der Vorgeschichte zu seiner Gründung in den späten 1960er und frühen 1970er Jahren bis zu den jüngsten Entwicklungen nach dem Inkrafttreten des Vertrags von Lissabon, stellte den ersten inhaltlichen Schwerpunkt der Konferenz

\section{The European Council - The Many Faces of a Key Institution}

Gemeinsame Tagung der Coelner Monnet Vereinigung für EU Studien e.V. (COMOS), Köln, des Instituts für Europäische Politik (IEP), Berlin, und der Trans European Policy Studies Association

(TEPSA), Brüssel

Mit Unterstützung der Hanns Martin Schleyer-Stiftung und der Landesvertretung Nordrhein-Westfalen in Brüssel

20.-21. Juni 2013, Brüssel

\section{Begrüßung}

Elfriede REGELSBERGER, Institut für Europäische Politik (IEP), Berlin

Die Entwicklung des Europäischen Rates

Vorsitz: Tobias KUNSTEIN, Universität zu Köln

Andrew DUFF, Mitglied des Europäischen Parlaments

Jean-Paul JACQUÉ, Trans European Policy Studies Association (TEPSA), Brüssel

Philippe DE SCHOUTHEETE, EGMONT Royal Institute for International Relations, Brüssel

Der Europäische Rat auf der Weltbühne

Vorsitz: Mathias JOPP, Institut für Europäische Politik (IEP), Berlin

Gianni BONVICINI, Istituto Affari Internazionali (IAI), Rom

* Dr. Tobias Kunstein, Institut für Politikwissenschaft und Europäische Fragen, Universität zu Köln. Wulf Reiners, M.A., Institut für Politikwissenschaft und Europäische Fragen, Universität zu Köln.

1 Eine Dokumentation der Tagung mit Fotos findet sich auch im Internet unter: http://www.jeanmonnetchair.unikoeln.de/27512.html (letzter Zugriff: 14.10.2013). 
dar. Die Referenten zeigten auf, wie die Staatsund Regierungschefs durch ihre Treffen in Den Haag (1969), Paris (1973) und Kopenhagen (1974) die potenzielle Tragweite europäischer Gipfel erkannten und zur dauerhaften Einrichtung des Europäischen Rates inspiriert wurden. Hierbei wurde diskutiert, inwieweit die Verstetigung der Treffen als Teil eines Kompromisses zwischen den Mitgliedstaaten angesehen werden kann, im Gegenzug zur Installation des intergouvernementalen Gremiums dem Europäischen Parlament das Recht der Direktwahl einzuräumen, um das demokratische Defizit in der Europäischen Union zu lindern. Insgesamt sei im Zeitverlauf eine zunehmende Formalisierung des Europäischen Rates und seiner Arbeitsweise zu beobachten, betrachte man die Entwicklung vom Inkrafttreten der Einheitlichen Europäischen Akte und des Vertrags von Maastricht, die dem Gremium keine spezifischen Aufgaben zugewiesen hatten, bis hin zu den Änderungen durch den Vertrag von Lissabon, der den Europäischen Rat zu einem Organ der Europäischen Union machte und mit einem hauptamtlichen Präsidenten ausstattete. Dabei wurde allerdings auch auf die Flexibilität des Systems hingewiesen: Der Präsident der Europäischen Zentralbank (EZB) sei in der Wirtschafts- und Finanzkrise zu einem QuasiMitglied des Europäischen Rates mit erheblichem Einfluss geworden.

Mit Blick auf den Aufbau der Institution wurde auch die Mitgliedschaft des Kommissionspräsidenten im Europäischen Rat kontrovers diskutiert. Obwohl sein Ausschluss von den Gipfeltreffen $\mathrm{zu}$ keinem Zeitpunkt in der Geschichte realistisch gewesen sei, könne die derzeitige Praxis nachteilig für die Kommission sein, wenn sich die Staats- und Regierungschefs weigerten, öffentlich die Verantwortung für gemeinsame Beschlüsse auf europäischer Ebene zu übernehmen. Dadurch könne die Kommission in eine Komplizenschaft für politische Entscheidungen gezogen werden, die teilweise nicht ihrer eigenen Auffassung entsprächen. So herrschte Einigkeit unter den Teilnehmern, dass die Rolle der Kommission als unabhängiger Kritiker der Zweckkompro-
Jim CLOOS, Rat der Europäischen Union, Brüssel

Geoffrey EDWARDS, Universität Cambridge

Grußworte anlässlich der Jubiläen von

Wolfgang Wessels

Hartmut MARHOLD, Centre international de formation européenne (CIFE), Nizza/Berlin, und Coelner Monnet Vereinigung für EU Studien e.V. (COMOS), Köln

Mathias JOPP, Institut für Europäische Politik (IEP), Berlin

Tischrede im Rahmen des gemeinsamen Abendessens

Luuk VAN MIDDELAAR, Mitglied des Kabinetts des Präsidenten des Europäischen Rates, Herman Van Rompuy, Brüssel

\section{Begrüßung}

Rainer STEFFENS, Leiter der Landesvertretung Nordrhein-Westfalen bei der EU, Brüssel

Der Europäische Rat als Wirtschaftsregierung der EU

Vorsitz: Tobias KUNSTEIN, Universität zu Köln

Iain BEGG, London School of Economics (LSE), London

Janis EMMANOUILIDIS, European Policy Centre (EPC), Brüssel

Gabriel GLÖCKLER, Europäische Zentralbank, Frankfurt Main

\section{Zusammenfassung der Tagungsergebnisse}

Philippe DE SCHOUTHEETE, EGMONT Royal Institute for International Relations, Brüssel

Jean-Paul JACQUÉ, Trans European Policy Studies Association (TEPSA), Brüssel

Wolfgang WESSELS, Universität zu Köln

misse im Europäischen Rat geschützt werden müsse.

Uneinig waren sich die Teilnehmer hingegen bezüglich der Rolle des Europäischen Rates in Abgrenzung zum Rat der Europäischen Union und als potenzieller Bestandteil der Legislative 
im EU-System. Die Debatte nahm ihren Anstoß an der Vielzahl von Beschlüssen, darunter auch zahlreiche Detailregelungen in der $\mathrm{Zu}-$ ständigkeit von Fachministern, die de facto durch den Europäischen Rat getroffen würden, obwohl ihm auf europäischer Ebene de jure die Legitimität und zuweilen auch die technische Expertise fehlten. In einer anderen Lesart erschien der Europäische Rat dagegen als die einzige legitimierte Instanz, die in Krisensituationen Entscheidungen von weitreichender Bedeutung treffen könne.

Kritische Töne wurden angesichts der zunehmenden ,Präsidentialisierung ' des EU-Systems laut, womit auf die Koexistenz der Präsidenten des Europäischen Rates, des Parlaments und der Kommission sowie der rotierenden Ratspräsidentschaft angespielt wurde. Diese Vielzahl sei insbesondere einer sinnvollen Außenvertretung der Europäischen Union hinderlich. In einer möglichen zukünftigen Fusion der Ämter des Präsidenten der Europäischen Kommission und des Präsidenten des Europäischen Rates wurde allerdings keine Lösung gesehen, da die Initiative im Rechtsetzungsprozess und das Aushandeln politischer Kompromisse zwei unvereinbare Funktionen darstellten. In diesem Zusammenhang kam auch zur Sprache, dass der Europäische Rat in jüngerer Zeit zunehmend das Initiativrecht der Kommission übernommen und letztere damit auf ihre Funktion als Hüterin der Verträge reduziert habe. Dieser These wurde entgegengehalten, dass die Kommission zumindest für die Länder der Eurozone in Bezug auf makroökonomische und Budgetfragen zusätzliche Kompetenzen und damit exekutive Macht hinzugewonnen hätte.

In der weiteren Diskussion über die Fortentwicklung des Europäischen Rates wurde die Bedeutung von Maßnahmen zur Stützung der Eurozone unterstrichen, die nicht alle aus dem Europäischen Rat hervorgegangen seien. Dazu gehörten insbesondere die Entscheidungen des in den Verträgen nicht vorgesehenen EuroGipfels. Es wurden darüber hinaus eine Reihe von Änderungsvorschlägen der sogenannten
Spinelli-Gruppe vorgestellt, die Ausgangspunkt für einen Konvent ab dem Jahr 2015 und einer anschließenden Vertragsrevision bilden könnten. $\mathrm{Zu}$ den Ideen zählt, den Präsidenten des Europäischen Rates zukünftig aus dem Kreis der Staats- und Regierungschefs zu wählen, um einerseits dem Amt ein stärkeres Profil zu verleihen und andererseits zu gewährleisten, dass der Makler eines Kompromisses diesen auch selbst umsetzen muss.

\section{Der Europäische Rat auf der Weltbühne}

Der zweite inhaltliche Block der Konferenz widmete sich der Rolle des Europäischen Rates auf internationaler Ebene. Die Debatte wurde unter der Prämisse geführt, dass es nicht der Europäische Rat sei, der global in Erscheinung treten müsse, sondern die Europäische Union als Ganzes, deren internationale Rolle der Europäische Rat mitzugestalten hätte.

Im Mittelpunkt der Diskussion standen zunächst die Auswirkungen der verschiedenen Neuerungen des Vertrags von Lissabon - etwa die Rechtspersönlichkeit der Europäischen Union, der hauptamtliche Präsident des Europäischen Rates oder der Europäische Auswärtige Dienst (EAD). Dabei überwogen eher skeptische Einschätzungen: Anders als zum Beispiel in den 1970er Jahren wollten die Staats- und Regierungschefs viele außenpolitische Fragen heute nicht mehr auf europäischer Ebene diskutieren. Auch mangele es sowohl der Gemeinsamen Außen- und Sicherheitspolitik (GASP) als auch der Gemeinsamen Sicherheits- und Verteidigungspolitik (GSVP) an klaren Prioritäten und (finanziellen) Ressourcen. Trotz der Lissabonner Innovationen hätte sich auf der Arbeitsebene fast nichts geändert - ungeachtet der flexiblen Möglichkeiten, die die Verträge zum Beispiel in Form der Ständigen Strukturierten Zusammenarbeit böten. Es fehle schlichtweg an politischem Willen, diese Möglichkeiten tatsächlich zu nutzen, um gemeinsame Projekte zu verwirklichen.

Lediglich die Einführung des hauptamtlichen Präsidenten des Europäischen Rates wurde von 
einigen Teilnehmern als positiv für die Sichtbarkeit der Europäischen Union auf internationaler Ebene gewertet. Dem wurde jedoch entgegengehalten, dass Überlappungen zwischen dem internationalen Mandat des Präsidenten des Europäischen Rates und der Hohen Vertreterin der Union für Außen- und Sicherheitspolitik einer stärkeren internationalen Rolle der Europäischen Union abträglich seien. In diesem Zusammenhang wurde darauf hingewiesen, dass der Präsident des Europäischen Rates Herman Van Rompuy sich mindestens einmal wöchentlich mit dem Präsidenten der Europäischen Kommission José Manuel Barroso treffe, aber nur im Monatsrhythmus mit der Hohen Vertreterin Catherine Ashton. Mit Blick auf den Posten der Letztgenannten äußerten zudem viele Teilnehmer Bedenken bezüglich ihres Aufgabenzuschnitts. Es hätte sich als praktisch unmöglich erwiesen, alle Rollen - von der EU-Außenbeauftragten bis zur VizePräsidentin der Kommission und der Leiterin des EAD - gleichzeitig befriedigend auszufüllen.

Zu den institutionell angelegten Schwierigkeiten gehöre darüber hinaus die Fragmentierung der Außenvertretung der Europäischen Union. Die Bereiche Handel, Entwicklung und die externen Aspekte von Gemeinschaftspolitiken seien in den Händen der Kommission, während für die Beziehungen mit strategischen Partnern, Erweiterung, Nachbarschaftspolitik und GASP weiterhin der Rat beziehungsweise der Europäische Rat maßgeblich sei. Als erster Schritt zur Verbesserung der Außenvertretung wurde vorgeschlagen, die Zahl und Länge von Stellungnahmen der Europäischen Union in internationalen Foren und bei den Schlussfolgerungen des Europäischen Rates zu reduzieren. Dass eine institutionelle Fortentwicklung auch in der Frage der Außenvertretung möglich sei, zeige das Beispiel G7/G8, in deren Rahmen die Kommission ihr Rederecht im Namen der Europäischen Union erst im Laufe der Zeit erlangt hätte. Bezüglich der Kommission wurde aber auch die Meinung geäußert, dass sie in der Außenvertretung der gemischten Gemeinschaftskompetenzen ,ihr Blatt überreizt“ habe. Als
Folge sei ihre Kompetenz in diesen Feldern von den Mitgliedstaaten auf die Bereiche der ausschließlichen Gemeinschaftskompetenz zurückgeführt worden.

Mehrfach wurde der Europäische Rat als Club der am stärksten legitimierten Vertreter der Mitgliedstaaten und somit als der beste Ort bezeichnet, um gemeinsame außenpolitische Entscheidungen zu treffen. Diese These werde dadurch gestützt, dass der weit überwiegende Teil europäischer Außenpolitik nach wie vor auf Ebene der Mitgliedstaaten angesiedelt sei. Allerdings müsse in Rechnung gestellt werden, dass das politische Überleben der Staats- und Regierungschefs im Europäischen Rat von den Wählern in ihren Heimatländern abhinge. Demnach sei es strukturell bedingt schwierig, im Europäischen Rat langfristige Strategien zu verfolgen. Ein aktuelles Beispiel für den möglichen Widerspruch zwischen nationaler und europäischer Perspektive sei das Vorgehen des britischen Premiers David Cameron, der einerseits mit dem US-amerikanischen Präsidenten Barack Obama über das Freihandelsabkommen zwischen den USA und der Europäischen Union verhandele, während er intern die Idee eines Referendums über die Zukunft der Beziehungen zwischen Großbritannien und der Europäischen Union vorantreibe.

Es wurde wiederholt darauf hingewiesen, dass es auf europäischer Ebene keine ,KompetenzKompetenz' gebe und in außenpolitischen Fragen nach wie vor fast immer Einstimmigkeit erforderlich sei. Deshalb sollten die Erwartungen an die europäische Ebene nicht zu hoch ausfallen. Als bedauerlich wurde angesehen, dass der Europäische Rat mit zunehmender Größe seine Funktion als ,espace de réflexion verloren hätte und sich heute jeweils 28 Mitglieder zu ein- und demselben Problem äußern wollten. Trotz dieser Einschränkungen würden die Mitgliedstaaten nach Einschätzung der Referenten den Europäischen Rat als sinnvolle Ergänzung ihres außenpolitischen Instrumentariums ansehen, zumal Sozialisationsprozesse innerhalb des Gremiums berücksichtigt werden müssten, die Unterschiede zwischen nationalen Positionen abschwächen könnten. 


\section{Der Europäische Rat als Wirtschaftsregierung}

Unter der Fragestellung, ob der Europäische Rat als ,Europäische Wirtschaftsregierung ‘ verstanden werden könne, widmete sich der dritte inhaltliche Schwerpunkt der Konferenz politökonomischen Fragestellungen. Die Referenten gingen dabei ausführlich auf das veränderte Zusammenspiel zwischen Europäischem Rat, EZB und Europäischer Kommission ein und machten deutlich, dass für eine Wirtschaftsregierung der politische Entscheidungsspielraum maßgeblich sei.

Die Debatte entwickelte sich aus der Einsicht der letzten Krisenjahre, dass Geldpolitik allein die Stabilität der gemeinsamen Währung nicht garantieren könne. Daraus folgte die Frage, ob eine Verknüpfung mit der Fiskalpolitik zwingend sei und welche Institution unter diesen Umständen überhaupt als Wirtschaftsregierung handeln könne. Einige Konferenzteilnehmer äußerten die Ansicht, dass die Europäische Kommission in ihrer derzeitigen Gestalt dafür nicht geeignet sei. Man könne von ihr nicht erwarten, dass sie Aufgaben außerhalb ihres Mandats übernimmt. Sie verfüge, etwa im Vergleich zur EZB, nicht über die notwendigen Instrumente, um die Krise wirkungsvoll zu bekämpfen. Zudem schrecke sie offensichtlich vor politisch schwierigen Entscheidungen zurück, wie im Fall Sloweniens und Spaniens, als keine Verfahren im Rahmen des, Sixpacks' eröffnet wurden. Die Kommission habe infolge ihrer zunehmenden Politisierung das Vertrauen der Mitgliedstaaten verloren und insgesamt eine Schwächung erlebt. Das von ihr hinterlassene Vakuum sei schließlich von anderen Institutionen gefüllt worden - von der EZB, dem Internationalen Währungsfonds (IWF) und nicht zuletzt vom Europäischen Rat.

Der Europäische Rat habe aber nicht nur gegenüber der Kommission eine stärkere Position eingenommen. So komme es ihm gegenüber dem - formell hauptsächlich verantwortlichen - Rat Wirtschaft und Finanzen (ECOFIN) zugute, dass der Europäische Rat sich auch mit strategischer wirtschaftspolitischer Planung befassen könne, die zum Aufgabenbereich einer echten Wirtschaftsregierung gehöre. Darüber hinaus sei der Europäische Rat in Ermangelung eines , europäischen Steuerzahlers ‘ der beste Ort, um eine Übernahme von Lasten durch die Europäische Union zu beschließen. Andererseits müsse eine Wirtschaftsregierung ,non-stop' arbeiten und nicht nur zu einigen wenigen Treffen im Jahr zusammentreten. Auch für Detailfragen der täglichen Koordination sei der Europäische Rat ungeeignet. Die Konferenzteilnehmer kritisierten in diesem Zusammenhang, dass der ECOFIN in der Krise $\mathrm{zu}$ viele derartige Fragen an die Staats- und Regierungschefs weitergegeben hätte.

Die Einführung der Euro-Gipfel wurde von den Konferenzteilnehmern hingegen als nützliche Entwicklung erachtet, wobei ihre Einberufung an den tatsächlichen Diskussionsbedarf gebunden werden sollte und nicht an einen festen Zeitplan. Einer Fortführung der bisherigen Praxis, den Europäischen Rat und den EuroGipfel von demselben Präsidenten leiten zu lassen, standen die Teilnehmer positiv gegenüber. Allerdings gaben sie zu bedenken, dass damit theoretisch Nichtmitglieder der Eurozone von einer Präsidentschaft des Europäischen Rates ausgeschlossen wären.

Nach Ansicht der Referenten befindet sich der Europäische Rat seit mehreren Jahren in einem ,Krisen-Modus', in dem der Exekutive naturgemäß eine stärkere Bedeutung zukommt. Insofern sei es wahrscheinlich, dass der Europäische Rat wieder an Bedeutung verlieren werde, sobald die Krise vorbei sei. Allerdings wurde die Führungsrolle des Europäischen Rates unter Hinweis auf die EZB auch bereits für die zurückliegenden Jahre relativiert. Diese habe seit dem Gipfel von Paris 2008 an Treffen des Europäischen Rates teilgenommen und als Agenda-Setter wie auch in der Umsetzung der komplizierten Folgeprozesse großen Einfluss gehabt. Die (Krisen-)Gipfel selbst gerieten zudem mitunter zu medialen Inszenierungen als Antwort auf die Forderungen der Märkte, wie die Beispiele der Van Rompuy-Taskforce und auch der Fiskalvertrag zeigten. 
Trotz dieser Entwicklungen bestand unter den Konferenzteilnehmern Konsens, dass eine Wirtschaftsregierung mit Durchgriffsrechten in der Europäischen Union gegenwärtig nicht gegeben sei, weder in Form des Europäischen Rates oder des Euro-Gipfels noch einer anderen Institution. In Ermangelung einer solchen Wirtschaftsregierung wurden als temporäre Lösungen bilaterale Verträge zwischen nationalen Regierungen und der Europäischen Union, wie im Rahmen des Europäischen Semesters, diskutiert. Diese wurden als aussichtsreiches Instrument angesehen, um die Selbstverpflichtungen der Mitgliedstaaten effektiver umzusetzen. Gleichzeitig war die vorherrschende Meinung, dass die Krisenmaßnahmen schon die Grenze dessen erreicht hätten, was der bestehende Rechtsrahmen der Europäischen Union erlaube.

\section{Der Europäische Rat - Schlüsselinstitution im politischen System der EU?}

Die gemeinsame Tagung der Coelner Monnet Vereinigung für EU Studien e.V. (COMOS), des Instituts für Europäische Politik (IEP) und der Trans European Policy Studies Association (TEPSA) lieferte eine präzise Betrachtung des Europäischen Rates in seiner historischen Entwicklung aus den Perspektiven von Wissenschaft und Praxis. Die einzelnen Beiträge verdeutlichten seine vielfältigen Rollen innerhalb des europäischen Integrationsprojekts - sei es in der Systementwicklung oder im Krisenmanagement, sei es in der Außen- oder Wirtschaftspolitik. Gleichzeitig wurde klar, dass der Europäische Rat nicht getrennt von den anderen Institutionen gesehen und verstanden werden kann. Nicht nur stellt er den Ausgangspunkt für Vertragsrevisionen und somit den Ort des Zuschnitts und der Umgestaltung der anderen Institutionen dar. Er ist auch so eng in das politische System eingebunden, dass eine
Änderung seines eigenen Charakters unweigerlich Auswirkungen auf die politischen Mitspieler und die institutionelle Balance des gesamten Systems hat.

Als ein wesentliches Ergebnis der Tagungsbeiträge und -diskussionen lässt sich festhalten, dass der Europäische Rat in der Tat als Schlüsselinstitution verstanden werden kann. Dennoch ist er kein Allheilmittel für die Lösung aller Probleme auf europäischer Ebene. So zeigte sich, dass der Europäische Rat zwar für manche Aufgaben prädestiniert ist, zum Beispiel als , deus ex machina“ im Fall von Krisen oder als Forum für Fragen von besonderer Tragweite. Gleichzeitig gibt es Aufgaben und Bereiche, die ihn überfordern können. Dazu gehören beispielsweise die regelmäßigen Aufgaben einer Wirtschaftsregierung oder Fachfragen, die einen hohen Grad an technischem Detailwissen erfordern. Auch bleiben Zweifel bezüglich der Legitimation seiner Entscheidungen auf Grundlage des aktuellen rechtlichen Rahmens.

Geht man von der Schwere einer Krise als Gradmesser für das Gewicht des Europäischen Rates als bestes Krisenwerkzeug der Europäischen Union aus, durchlebt der Europäische Rat derzeit womöglich eine Phase, in der er wichtiger und stärker im EU-System ist als je zuvor in der Geschichte. Vor diesem Hintergrund schien die Konferenz im Juni 2013 ebenso gut terminiert wie zugeschnitten. Die Eigenschaften des Europäischen Rates wie Zusammensetzung, Themenspektrum und Einfluss seiner Entscheidungen machen ihn zu einem faszinierenden Forschungsgegenstand auch außerhalb von Krisenzeiten. Es darf davon ausgegangen werden, dass Wolfgang Wessels diese Einschätzung teilt und dass die Relevanz des Themas in weiteren Arbeiten des Jubilars zum Ausdruck kommen wird. 